\title{
Pause machen
}

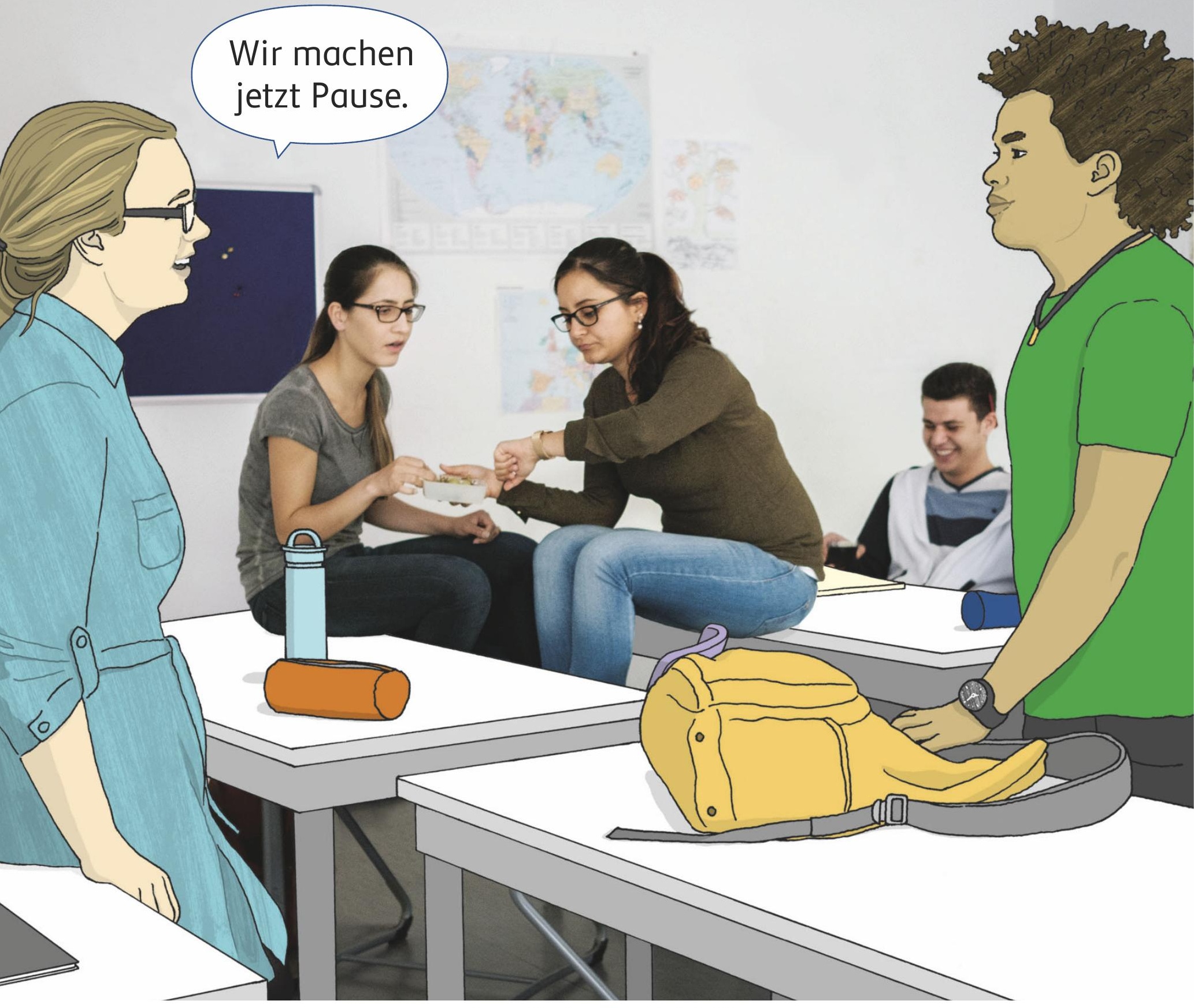

1 Was siehst du? Sprich. 8

2 Höre und sprich nach. (2) (-)

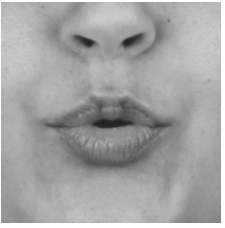

\section{Pause}

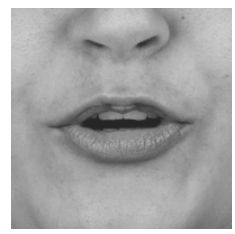

\section{Bäume}




\section{Pause machen}

3a Sprich nach und fahre mit dem Finger nach.

\section{(2) (1) 7}

(3) 4
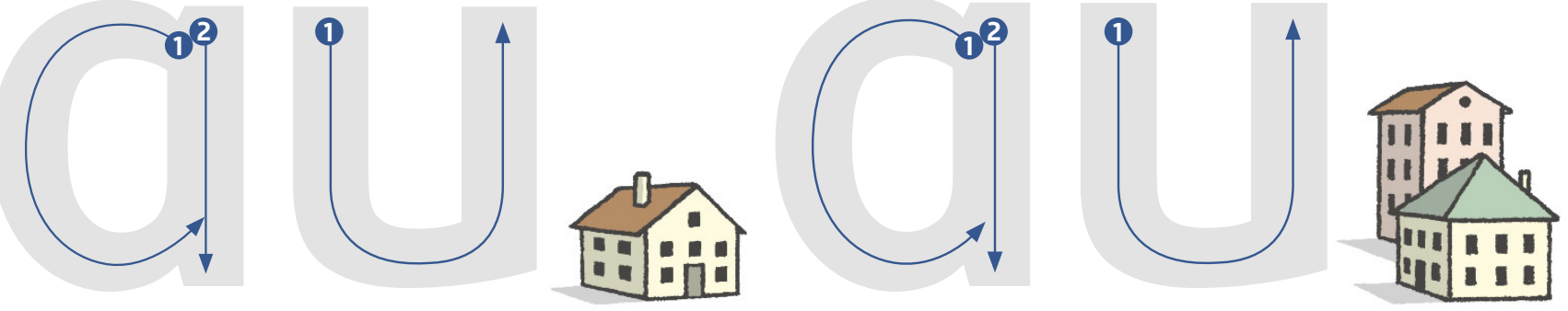

3b Schreibe.

Au

au

\section{das Haus}

\section{äu}

\section{die Häuser}

3c Schreibe Au au äu oder das ganze Wort nach.

$\begin{array}{llll}\text { das Auto } & \text { das Haus } & \text { der Bauch } & \text { der Raum } \\ \text { das Auge } & \text { die Häuser } & \text { draußen } & \text { der Baum } \\ \text { die Pause } & \text { die Räume } & \text { kaufen } & \text { die Bäume }\end{array}$

4 Wie viele Silben hat das Wort? Höre und kreuze an.

(2) (9)

Pause

Kaufhäuser

$2 \quad 3 \quad 4$ 
5a Ordne zu und schreibe.

der Supermarkt das Restaurant das Kaufhaus das Café
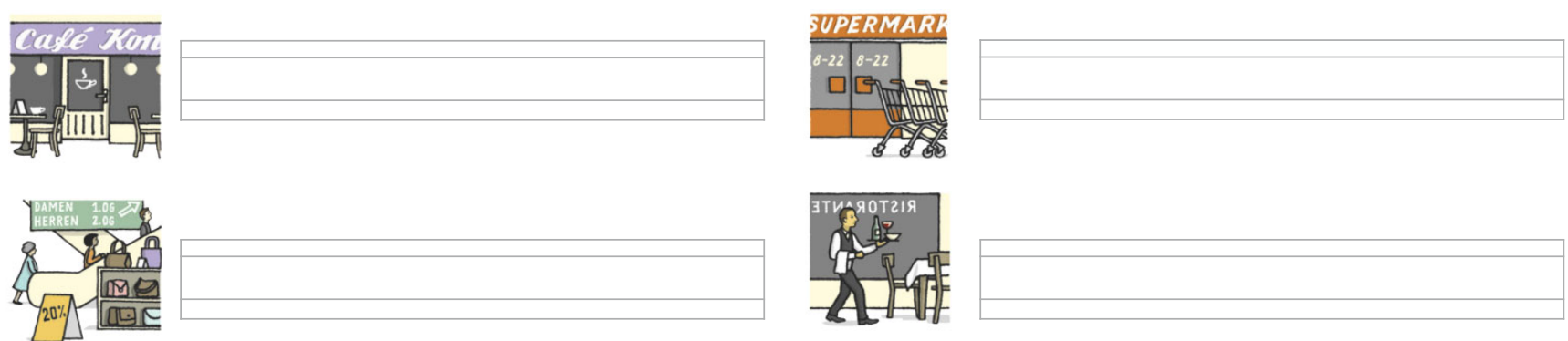

5b Lies und markiere die Nomen. 目

Hallo, Leute. Ich bin Nariman.

Heute kaufe ich viele Dinge:

brauchen, haben, kaufen + Akkusativ

der - den ein - einen

die - die eine - eine

das-das ein - ein

Ich brauche einen Stift

eine Orange 0 , eine Limo 6 , eine Pizza $\$$, ein Wasser 8 ,

einen Kaffee 3 , eine Hose W und einen Kuchen

5c Was passt zusammen? Verbinde.

Wo gibt es ...

... einen Stift? Im Supermarkt.

... eine Orange? Im Kaufhaus.

... eine Limo?

... eine Pizza?

... einen Kaffee?

Im Café.

Im Restaurant.

... eine Hose?

... einen Kuchen? 


\section{Pause machen}

6a Sprich und zeige mit deiner Hand.

Ich mache eine Pause. .....•

Wir machen eine Pause. $\ldots . . . \bullet^{\bullet}$

Du machst Hausaufgaben. . .•・..

Ihr macht Hausaufgaben. ..•...

Er braucht einen Stift.

Sie brauchen einen Stift. . ..... $\bullet^{\bullet}$

6b Mache für jede Silbe einen Schritt nach vorne.

7 Wer braucht was? Schreibe.

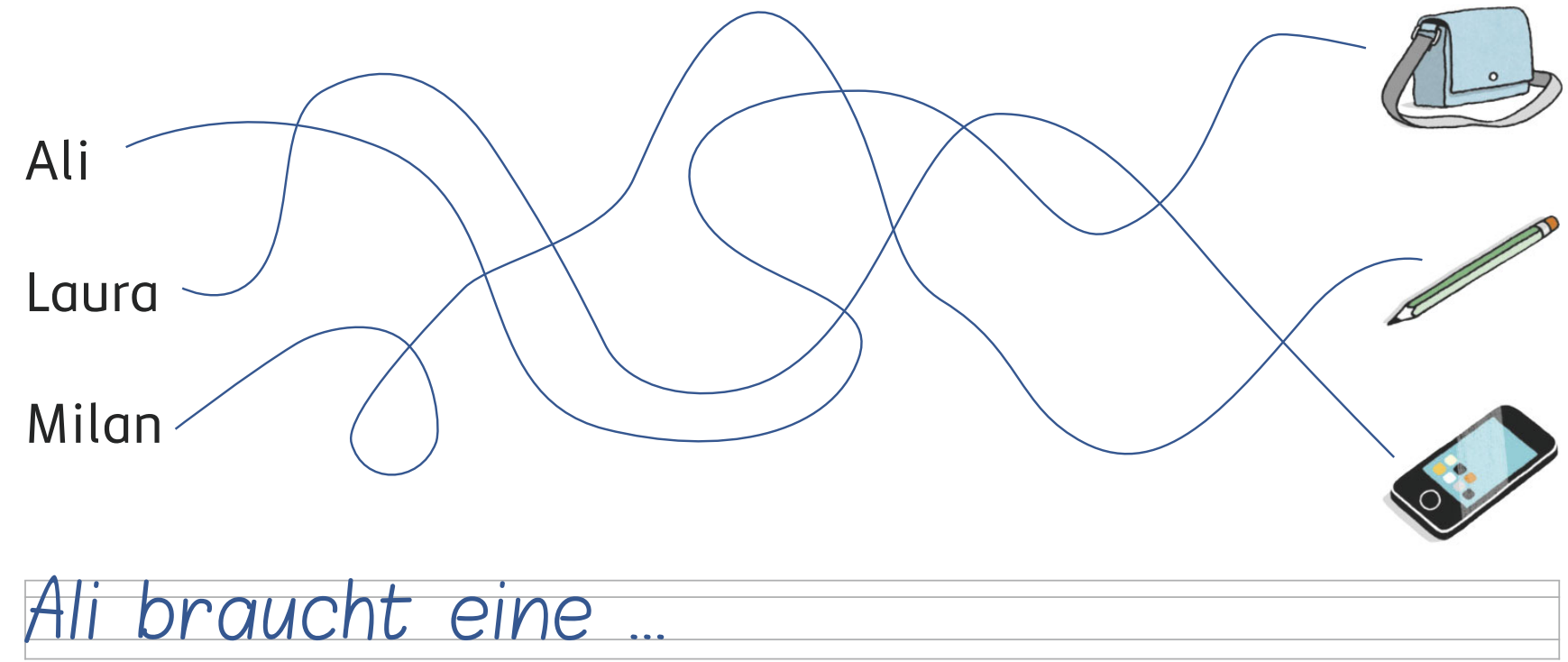

Ali braucht eine 


\section{au äu}

8 Lies und markiere au. 自

Dieses Haus ist unsere Schule.

Wir haben Deutsch in Raum Nummer 205.

In Raum 18A machen wir unsere Hausaufgaben.

Es ist 10.30 Uhr. Jetzt machen wir Pause.

9a Markiere a e i o u und au äu ei.

Sprich und mache die Handzeichen dazu.

(2) 8

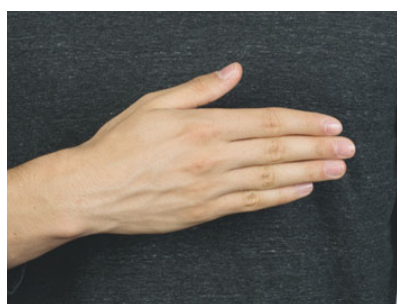

a e i o u

die Hand

die Polizei

die Milch

die Räume der Reis

die Tomate

die Hausaufgabe

die Häuser

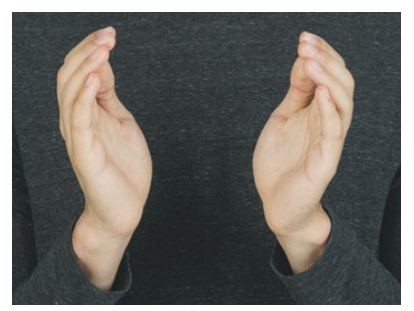

au äu ei

der Elefant

das Datum

das Haus

die Pause

9b Was hörst du? Höre und kreuze an. (2) (1),

$\begin{array}{lllllllll}\text { ei } & \text { au } & \text { äu } & \text { ei } & \text { au } & \text { äu } & \text { ei } & \text { au } & \text { äu } \\ & \square & \square & \square & \square & \square & \square & \square & \square\end{array}$




\section{Pause machen}

10a Lies die Sätze. Markiere die Verben. 目

Dieses Haus ist unsere Schule.

Sie heißt Kant-Schule.

Sie hat zehn Räume.

In Raum Nummer 205 lernen wir Deutsch.

Und hier können wir Hausaufgaben machen.

10b Konjugiere die Verben.

\begin{tabular}{|c|c|c|c|}
\hline & lernen & machen & heißen \\
\hline ich & & & \\
\hline du & & & \\
\hline er/sie/€ & & & \\
\hline wir & & & \\
\hline ihr & & & \\
\hline sie/Sie & & & \\
\hline
\end{tabular}

10c Eine Person liest den Text aus 10a, die andere Person schreibt. Dann Wechsel. 


\section{au äu}

11a Höre und lies mit. (2) (1010

Hallo.

Ich bin Tung Nguyen.

Ich wohne in Augsburg.

Ich lerne viel Deutsch.

Am Vormittag bin ich in

der Schule. Am Nachmittag

mache ich meine

Hausaufgaben.

11b Meine Aufgabe für dich. Was ist das? Schreibe das Wort.
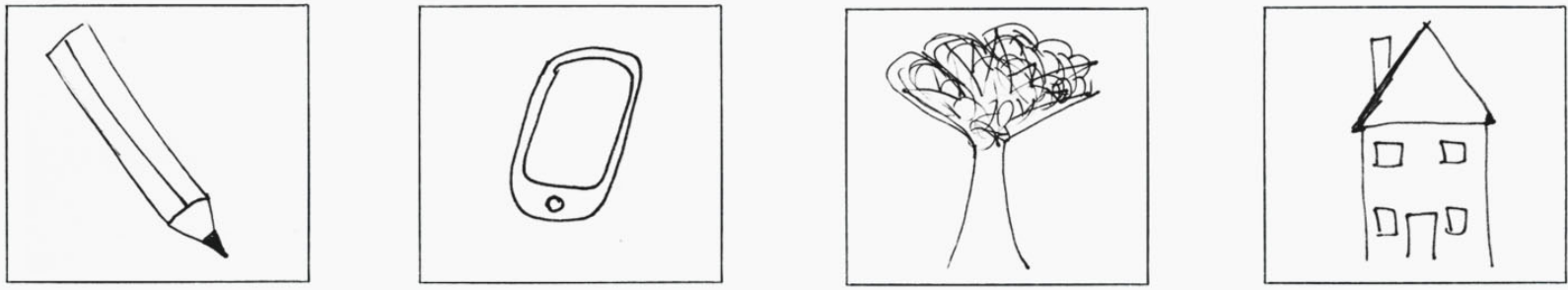

der Stift

11c Schreibe eine eigene Aufgabe. 
$\star$ Schreibe die Personalpronomen.

Ich mache eine Pause. machen eine Pause.

machst eine Pause. macht eine Pause.

$\star \star$ Wo kaufst du das? Schreibe.

die Tomate die Banane das Handy Im Café: der Kuchen die Limo die Suppe der Kaffee der Tee das Kleid die Schokolade die Hose die Nudeln

der Kuchen 
$\star$ Singular und Plural. Verbinde.

der Raum

das Haus

der Baum

der Bauch

die Frau

das Auto

die Hausaufgabe die Bäume

die Hausaufgaben

die Autos

die Frauen

die Bäuche

die Häuser

die Räume

$\star \star$ Welches Verb passt? Schreibe.

lernen machen kaufen haben wohnen

Das Kind ein Eis.

Wo

du?

Ich

Deutsch.

Die Schule zehn Räume.

Hier wir Pause.

$\star \star \star$ Schreibe den unbestimmten Artikel.

Ich kaufe Limo.

Wir machen

Pause.

Dana hat

Buch.

Ihr kauft

Hund.

Amir hat

Haus.

Sie brauchen

Auto. 
Schreibe.

\section{das Haus, die Häuser}

die Hausaufgabe, die Hausaufgaben

die Pause, die Pausen

der Pausenhof, die Pausenhöfe

der Raum, die Räume

dieser, diese, dieses

Lies und sprich.

Es ist laut.

Wir machen

Ich brauche einen Stift. Pause.

Ich kaufe einen Stift im Supermarkt. 


\section{Lernen unterwegs}

Singular und Plural.

Kombiniere die Wortpaare.

der Aufzug

die Aufzüge

das Haus

die Häuser

das Auge

die Augen

das Auto

die Autos

die Maus

die Mäuse der Baum

die Bäume

der Raum

die Räume

die Pause

die Pausen

die Hausaufgabe

die Hausaufgaben

der Bauch

die Bäuche der Staubsauger

die Staubsauger

das Kaufhaus

die Kaufhäuser

der Supermarkt

die Supermärkte

der Buchstabe

die Buchstaben

der Mann

die Männer 
Open Access Dieses Kapitel wird unter der Creative Commons Namensnennung - Nicht kommerziell - Keine Bearbeitung 4.0 International Lizenz (http://creativecommons.org/licenses/by-nc-nd/4.0/deed.de) veröffentlicht, welche die nicht-kommerzielle Nutzung, Vervielfältigung, Verbreitung und Wiedergabe in jeglichem Medium und Format erlaubt, sofern Sie den/die ursprünglichen Autor(en) und die Quelle ordnungsgemäß nennen, einen Link zur Creative Commons Lizenz beifügen und angeben, ob Änderungen vorgenommen wurden. Die Lizenz gibt Ihnen nicht das Recht, bearbeitete oder sonst wie umgestaltete Fassungen dieses Werkes zu verbreiten oder öffentlich wiederzugeben.

Die in diesem Kapitel enthaltenen Bilder und sonstiges Drittmaterial unterliegen ebenfalls der genannten Creative Commons Lizenz, sofern sich aus der Abbildungslegende nichts anderes ergibt. Sofern das betreffende Material nicht unter der genannten Creative Commons Lizenz steht und die betreffende Handlung nicht nach gesetzlichen Vorschriften erlaubt ist, ist auch für die oben aufgeführten nicht-kommerziellen Weiterverwendungen des Materials die Einwilligung des jeweiligen Rechteinhabers einzuholen. 